\title{
“Ejercicio y pulmón” ¿Qué hemos aprendido en las jornadas de otoño 2012?
}

\author{
2012 Fall Meeting: what do we have learned about physical exercise and the lung?
}

Las recientes Jornadas de Otoño de la Sociedad Chilena de Enfermedades Respiratorias en su $31^{a}$ versión abordaron un tema nunca antes tratado en encuentros de este tipo como fue "Ejercicio y Pulmón”, que permitió por su naturaleza, convocar a una amplia gama de especialistas, todos connotados en sus respectivas áreas de trabajo, tanto de la neumología (medicina respiratoria y pediatría), cirugía de tórax, kinesiología, medicina del deporte y fisiología del ejercicio. Esto permitió congregar no solo a médicos sino también a kinesiólogos, profesores de educación física y de fisiología del ejercicio, enfermeras, tecnólogos-médicos y profesionales en formación. El resultado fue el esperado: más de 200 asistentes en el nuevo Centro de Convenciones de Rosa Agustina en Olmué, V Región y una participación muy activa en cada una de las sesiones interactivas.

Los temas tratados durante la jornada del viernes 30 Marzo y mañana del sábado 31 se enfocaron principalmente hacia la fisiología del ejercicio para comprender las modalidades de entrenamiento y rehabilitación, tanto en el sano deportista como en el paciente con patología cardiorrespiratoria; el test cardiopulmonar (TCP) como herramienta para precisar el estado funcional, la capacidad aeróbica, tanto en sanos como enfermos y sus proyecciones diagnósticas y pronósticas. Se discutieron los nuevos algoritmos para la evaluación del paciente candidato a cirugía resectiva pulmonar y la utilidad del TCP al respecto. Por último se presentaron las bases para comprender los beneficios del deporte, la seguridad de la práctica deportiva, su vinculación con el estado nutricional y las modalidades de entrenamiento que generan un mayor impacto en el control del peso.

La razón para elegir este tema está directamente vinculada a la contingencia nacional en lo que se refiere a factores de riesgo y estilos de vida, ilustrados en la Encuesta Nacional de Salud 2010 (www. minsal.cl) y los proyectos de trabajo 2011-2020 para nuestra población. La Encuesta Nacional de Salud informa de cifras realmente preocupantes tales como: sedentarismo en tiempo libre del 88\%, tabaquismo de $41 \%$, sobrepeso y obesidad de $67 \%$. Todo lo anterior incide en la prevalencia de condiciones que traducen graves riesgos para nuestra población como son: Hipertensión Arterial (27\%), Diabetes (9\%) y Depresión (17\%). ¿Cómo hacer frente a factores de riesgo adquiridos en nuestra población desde tan temprana edad y que ponen en riesgo los proyectos de vida de la población? ¿Cómo generar una fuente de cambio y un aporte concreto a la salud de nuestra comunidad al favorecer hábitos de vida saludable? El ilustrar, comunicar y generar conciencia es un paso, entre tantos que podemos dar para incidir en nuestro radio de acción motivando, una práctica deportiva regular, dieta saludable y abandono del hábito tabáquico. Trabajando en esos tres elementos sin duda estaremos contribuyendo en atenuar la frecuencia de las entidades más prevalentes y letales a nivel nacional como lo son la cardiopatía coronaria, la EPOC y el cáncer de pulmón.

Estas Jornadas incluyeron algunos cambios que las coordinadoras creemos deberán ser sostenidos en el tiempo en futuras instancias de encuentro como son: el llamado a valorar a nuestros pares, congregando solo especialistas nacionales, muchos de ellos profesores universitarios con magister o doctorado en sus respectivas disciplinas; el permitir el desarrollo de actividades vinculadas a la temática de las jornadas ofreciendo a los participantes la oportunidad de efectuar actividades deportivas durante el certamen y la generación de un impreso de resúmenes con extractos de las presentaciones y material de apoyo (aportado por los conferencistas). Todas las iniciativas anteriormente mencionadas hicieron de este encuentro una instancia singular de gran impacto en nuestra práctica médica diaria.

A continuación se presenta un resumen muy sucinto de las principales conferencias que se presentaron durante las Jornadas, para provocar vuestro interés y motivación a profundizar en cada una de estas temáticas. 


\section{Medicina respiratoria}

\section{Entrenamiento físico: Ajustes cardiovasculares, musculares y ventilatorios ${ }^{1-3}$}

Respuesta cardiovascular. En el entrenamiento aeróbico se produce disminución de la frecuencia cardíaca de reposo y sub-máxima (por predominancia del sistema parasimpático), aumento de volumen de cavidades cardiacas, aumento del grosor de las paredes, aumento del volumen por latido y de la perfusión miocárdica. El gasto cardíaco aumenta 6-7 veces sobre el de reposo en deportistas de elite comparados con sedentarios en los que aumenta sólo 4 veces. En el entrenamiento anaeróbico debido al efecto Valsalva que determina menor precarga por menor volumen diastólico final, una mayor postcarga por aumento de la presión arterial, aumento del inotropismo por activación simpática, genera un aumento más discreto del gasto cardíaco.

Repuesta muscular. A contar de la séptima semana post-entrenamiento anaeróbico, la hipertrofia muscular es evidente pese a que los cambios bioquímicos ocurrieron con anterioridad: aumento de PI3k (phosphatidylinositol-3 kinase), AKT1 (serine/threonine protein kinase), inhibición de Fox-O (Familia del factor de transcripción O), IGF-1(Insulin-like growth factor 1), MTOR (mammalian target of rapamycin), y disminución de la fosforilación de eEF2 (Eukaryotic translation elongation factor 2). Estos cambios determinan aumento de la síntesis proteica y reducción de la degradación. La hipertrofia muscular es mayor en las fibras rápidas que en las lentas. El estímulo aeróbico repetitivo genera síntesis mitocondrial con aumento de enzimas oxidativas determinando una mejoría en la capacidad de la fibra muscular oxidativa (tipo 1). Si se estimulan repetidamente las fibras rápidas, tipo 2, éstas se transforman en tipo 1 por activación de calcineurina y MEF-2 (myocyte enhancer factor-2).

Con el entrenamiento también se generan cambios en la microcirculación del músculo esquelético que aumentan la extracción de oxígeno.

Respuesta respiratoria. Hay aumento del trabajo respiratorio y de la frecuencia respiratoria y cardíaca. La ventilación minuto aumenta hasta 9 veces, mientras la relación volumen del espacio muerto/ volumen corriente (VD/VT) disminuye de 0,3 a 0,18, la frecuencia respiratoria aumenta 2 a 3 veces, el volumen corriente aumenta 5 veces y el cuociente respiratorio o RER aumenta de 0,8 a 1,1 -1,3. La difusión y transporte de gases se triplica por aumento de la superficie de intercambio, mayor volumen alveolar y reclutamiento asociado a la distensión de capilares pulmonares. La curva de disociación de oxígeno con la hemoglobina se desplaza a la derecha, la extracción de $\mathrm{O}_{2}$ (diferencia arterio-venosa) está aumentada debido a un mayor consumo de oxígeno por parte de las células musculares activas. $\mathrm{La} \mathrm{PaO}_{2}$ mejora manteniendo una saturación estable. Considerando los cambios cardiovasculares y musculares, se produce un aumento del consumo de $\mathrm{O}_{2}$ y producción de $\mathrm{CO}_{2}$.

\section{Índices cardiorespiratorios en deportistas de elite}

Los índices cardiorespiratorios se monitorizan constantemente para evaluar los progresos en el tratamiento y el rendimiento deportivo que finalmente depende de la capacidad de la musculatura de transportar el oxígeno y aportar la energía para la acción muscular. El consumo máximo de $\mathrm{O}_{2}\left(\mathrm{VO}_{2}\right.$ max) es uno de los índices capaces de integrar los componentes cardiocirculatorio, respiratorio y muscular. En general los corredores de fondo tienen niveles de $\mathrm{VO}_{2}$ max por sobre $70 \mathrm{~mL} / \mathrm{kg} / \mathrm{min}$ en comparación con un adulto sano sedentario que bordea los $40 \mathrm{~mL} / \mathrm{kg} / \mathrm{min}$. El $\mathrm{VO}_{2}$ max se consigue de acuerdo a la ecuación de Fick con un elevado gasto cardiaco y diferencia arterio-venosa de $\mathrm{O}_{2}$. El gasto cardiaco de un atleta podría ser de unos $36 \mathrm{~L} / \mathrm{min}$ y la diferencia arterio-venosa de $\mathrm{O}_{2}$ de al menos $15 \mathrm{~mL} / \mathrm{dL}$ y una extracción del 60\%. Desde el punto de vista metabólico los cuatro factores que deben ser modificados por el entrenamiento para aumentar el rendimiento aeróbico son: el transporte de $\mathrm{O}_{2}$, la extracción, la eficiencia mecánica y la limitación de la fatiga de los músculos respiratorios. Un alto rendimiento deportivo es el resultado de una adecuada carga genética y de sistemas de entrenamiento orientados a maximizar la capacidad de ejercicio.

\section{Rehabilitación respiratoria}

Las enfermedades respiratorias alteran la musculatura esquelética y respiratoria y modifican la mecánica ventilatoria generando una menor tolerancia al esfuerzo, en tales circunstancias el entrenamiento físico permite mejorar la capacidad de realizar ejercicio, reduciendo la disnea y mejorando la 
calidad de vida. La rehabilitación respiratoria según la ATS/ERS es la intervención multidisciplinaria, integrativa y basada en la evidencia, dirigida a pacientes con enfermedades respiratorias crónicas sintomáticas y con disminución de las actividades de la vida diaria. Está diseñada para reducir los síntomas, optimizar el estado funcional e independencia, aumentar la participación social y reducir los gastos en salud. Debe ser multidisciplinaria, individualizada y debe incorporar programas de entrenamiento físico, educación, nutrición y apoyo psicológico. El entrenamiento se basa en actuar sobre tres elementos: la musculatura respiratoria, trabajo aeróbico y de fuerza (anaeróbico). El entrenamiento permite mejorar la capacidad de realizar trabajo, reduciendo la disnea y mejorando la calidad de vida, pero no mejora necesariamente la función pulmonar. El entrenamiento aeróbico interválico mejora el $V E F_{1}$ y la resistencia. Se han obtenido resultados interesantes con entrenamientos de 30 minutos diarios con cargas del orden del 50\% de la presión inspiratoria máxima.

\section{Entrenamiento físico}

En el entrenamiento físico generalmente se incluye: fuerza, resistencia muscular, resistencia cardiovascular, equilibrio, coordinación y flexibilidad. Este debe ser de intensidad alrededor de $60-70 \%$ del $\mathrm{VO}_{2}$ max, idealmente a intervalos. Los principales efectos del entrenamiento son los siguientes: a) aumento del número de mitocondrias y de capilares musculares; b) mejoría en la perfusión en zonas altas del pulmón; c) mejoría en la relación ventilación/perfusión del pulmón; d) hipertrofia cardiaca, aumento del volumen sistólico y aumento del gasto cardiaco.

Es importante distinguir el efecto diferente que provoca un entrenamiento físico de resistencia respecto al de fuerza: el de resistencia (v.gr. ciclismo) induce mayor biogénesis mitocondrial aumentando la capacidad aeróbica; en cambio el de fuerza (v.gr. cargas, pesas) disminuye la fosforilación de eEF2 (Eukaryotic translation elongation factor 2) estimulando mayor síntesis proteica y por otro lado promueve que AKT1 (serine/threonine protein kinase) inhiba a Fox O (Familia de factor de transcripción O) y de esa manera inhibe la degradación proteica aumentando la masa muscular.

\section{Modalidad de entrenamiento más efectivo para el control del peso}

Desde el descubrimiento de la Ghrelina en 1999 (hormona "del apetito", sintetizada en el estómago), se ha estudiado su relación con el ejercicio y desde el año 2007 se sabe que realizar ejercicio previo a la ingesta calórica determina un menor apetito. El ejercicio de alta intensidad (anaeróbico) favorecería la oxidación de grasas debido a la activación de una kinasa dependiente de AMP que es la AMPK (AMP-activated protein kinase) que es un sensor metabólico, que desvía la oxidación de carbohidratos hacia la oxidación de grasas en el músculo esquelético debido a la inhibición de la acetil-Coenzima A-carboxilasa.

Un ejercicio que genera un mayor gasto calórico y mayor oxidación de las grasas es aquel que se efectúa en ayunas, sin ingesta de carbohidratos durante o después de la práctica, con una frecuencia de al menos 3 veces por semana (mejor aun 5 veces por semana), con duración > 45 minutos, a intervalos por sobre el ejercicio continuo, de alta intensidad inicial para terminar con baja intensidad y a temperaturas más bajas de la temperatura neutral (ya que aumenta la grasa parda que es altamente metabólica). Los beneficios del ejercicio a intervalos se explica porque genera una disminución del glicógeno intramuscular en el primer episodio de ejercicio favoreciendo la oxidación de grasas en los episodios posteriores, evita la disminución de la liberación de catecolaminas que se observa en el ejercicio continuo, produce una disminución más acentuada de los niveles de insulina plasmática posiblemente a causa de la mantención de la estimulación adrenérgica y aumenta la producción muscular de interleuquina-6 que provoca mayor liberación de GH (hormona del crecimiento) y cortisol, hormonas lipolíticas. Por otra parte, la ingesta de carbohidratos antes, durante o después del ejercicio produce el efecto contrario, inhibiendo la oxidación de grasas.

\section{Ejercicio, genética y ambiente}

El dogma del deportista de elite, genéticamente predeterminado ha sido desplazado por los sorprendentes hallazgos que revelan que el ejercicio es capaz de provocar cambios en las señales relacionadas con la síntesis proteica e incluso hasta en una base que puede provocar cambios en nuestra genética (zonas silentes del genoma) y esta variabilidad se expresaría en el fenotipo. Esto es porque somos producto de sistemas genéticos dinámicos. 


\section{Pruebas que evalúan capacidad funcional}

Dentro de los exámenes que evalúan capacidad funcional cabe destacar la caminata de 6 minutos, por su alta reproducibilidad, bajo riesgo y costo y el Test Cardiopulmonar, considerado el "gold standard" de la evaluación de capacidad funcional.

En la prueba de caminata de 6 min se han encontrado valores absolutos que discriminan mortalidad como es el caso de 332 m para Hipertensión pulmonar o de 350 m para EPOC. También se ha podido precisar la mínima diferencia en metros caminados que es relevante del punto de vista clínico, que por ejemplo en EPOC es de alrededor de $28 \mathrm{~m}$.

\section{Test cardiopulmonar (TCP) $)^{1-3}$}

Es una moderna herramienta para la evaluación integrada de la funcionalidad cardiorespiratoria y muscular. Permite evaluar la respuesta al ejercicio, la capacidad funcional aeróbica. Es un procedimiento seguro y preciso. Está disponible en muchos centros en el país, pero es subutilizado. Los valores normales de sus principales variables son los siguientes: $\mathrm{VO}_{2}$ peak: $>80 \%$ del valor predeterminado; umbral anaeróbico (UA) > 45\% $\mathrm{VO}_{2}$ max predeterminado; VEmax/VEmax predeterminada > 0,75; Reserva ventilatoria: Pulso de $\mathrm{O}_{2}>80 \%$ valor predeterminado; Reserva cardiaca $<15$ latidos/min.

\section{Definiciones}

$\mathrm{VO}_{2}$ : cantidad de $\mathrm{O}_{2}$ que el organismo consume por unidad de tiempo y refleja el nivel metabólico del individuo. En reposo es de $250 \mathrm{~L} / \mathrm{min}$. Se relaciona linealmente con la intensidad del ejercicio. El $\mathrm{VO}_{2}$ max informa sobre la tolerancia al esfuerzo.

UA: umbral anaeróbico es el valor del $\mathrm{VO}_{2}$ a partir del cual se produce un incremento en la concentración de ácido láctico debido a que el nivel de esfuerzo requiere de metabolismo anaeróbico (glucolisis) como vía productora de energía.

El TCP permite evaluar la capacidad aeróbica tanto en personas sanas, como deportistas de alto rendimiento y enfermos de patología cardíaca, respiratoria o muscular. El examen se realiza mediante un cicloergómetro-espirómetro, o a través de una trotadora (treadmill conectada al espirómetro) y el paciente efectúa un esfuerzo incremental máximo mientras se monitorizan variables oxihemodinámicas, cardiocirculatorias y ventilatorias de manera no invasiva.

Este examen logra de manera muy precisa informar sobre el funcionamiento integrado del sistema cardiovascular, respiratorio y muscular en condiciones dinámicas, mientras se efectúa un esfuerzo físico máximo, lo que genera información de la que no se dispone en las clásicas evaluaciones funcionales estáticas (espirometría, difusión, volúmenes y capacidades pulmonares). La exigencia de este test es máxima a diferencia del test de caminata de 6 min que es regulado por el propio paciente, no siendo exigido por el operador. Por definición es un test de esfuerzo incremental máximo. El paciente desarrolla un esfuerzo a cargas crecientes (medidas en watts) y mediante una mascarilla facial hermética es factible la medición del estado funcional respiratorio, la medición de flujos, el análisis de gases inspirados y espirados y sumado a las variables hemodinámicas que se monitorizan segundo a segundo de manera no invasiva, es posible la obtención de información tan relevante como la referente al consumo de oxígeno, umbral láctico, reserva cardiorrespiratoria entre otras variables (Tabla 1).

A través de dichas mediciones, el TCP permite objetivar la tolerancia al esfuerzo, el nivel o capacidad aeróbica del sujeto estudiado y ello es información relevante tanto para personas sanas no entrenadas, como para deportistas de alto nivel que deseen evaluar su nivel de entrenamiento y para pacientes con enfermedades cardíacas, pulmonares o neuromusculares. De manera muy precisa, este test da cuenta del estado funcional de al menos cuatro componentes: cardíaco, respiratorio, muscular y neurológico, permitiendo evaluar en forma dinámica las causas por las cuales se presenta dificultad respiratoria, intolerancia al esfuerzo, fatiga o cansancio, que ocasionalmente no son detectadas en aquellos exámenes funcionales convencionales, que se efectúan en condiciones estáticas.

Patrón cardiaco: $\mathrm{VO}_{2}$ peak disminuido, umbral anaeróbico (UA) disminuido, $\mathrm{VO}_{2} /$ carga disminuido, reserva respiratoria normal, reserva cardíaca disminuida, pulso de $\mathrm{O}_{2}$ disminuido, saturación normal, $\mathrm{VE} / \mathrm{VCO}_{2}$ puede estar aumentado. 
Patrón respiratorio: $\mathrm{VO}_{2}$ peak disminuido, UA normal o disminuido. $\mathrm{VO}_{2} / \mathrm{carga}$ normal, pulso de $\mathrm{O}_{2}$ puede estar disminuido o normal, disminución de la reserva respiratoria y saturación, y VE/VCO aumentado.

Las principales indicaciones del TCP son las siguientes: a) Evaluación de deportistas de alto rendimiento (nivel de entrenamiento, capacidad aeróbica y eventual modificación en el nivel trabajo); b) Disnea de origen no explicado o de origen incierto; c) En el sujeto sano antes de la programación de un programa de entrenamiento; d) Enfermedad respiratoria crónica (enfisema, fibrosis pulmonar, fibrosis quística, hipertensión pulmonar); e) Enfermedad cardíaca o respiratoria avanzada; f) Evaluación para programa de trasplante; g) Prescripción de rehabilitación; h) Evaluación preoperatoria de cirugía resectiva pulmonar por cáncer o patología estructural benigna (quistes, bronquiectasias), y cirugía de reducción del volumen pulmonar.

Las condiciones que contraindican el TCP son relativamente escasas destacándose entre ellas las siguientes: a) Incapacidad neuropsicológica para comprender instrucciones; b) Impedimento osteoarticular para efectuar la maniobra en cicloergómetro o el desconocimiento absoluto de la maniobra, c) Infarto agudo de miocardio o accidente cerebrovascular reciente (3 meses previos); d) Angina inestable; e) Arritmias no controladas; f) Hipertensión pulmonar inestable, con síncopes o arritmias no controladas, g) Disección aórtica; h) Embarazo.

Desarrollo del examen. Los pacientes deben acudir con sus exámenes de laboratorio, con el listado de medicamentos en uso, que no deben omitir el día del examen, además de vestuario cómodo, ropa de cambio y zapatillas. Deben estar con ayuno de 3-4 horas, sin fumar y evitar efectuar ejercicio físico intenso el día del examen. El examen dura alrededor de 30 minutos, dependiendo de las evaluaciones que sean pertinentes de efectuar en reposo (pequeña entrevista de síntomas y capacidad funcional y

Tabla 1. Principales variables evaluadas en la prueba cardiopulmonar y su significado

\begin{tabular}{|c|c|c|}
\hline Generales & Variables monitorizadas & Utilidad \\
\hline Objetivas & $\begin{array}{l}\text { Presión arterial, frecuencia cardíaca, } \\
\text { ECG continuo, Saturación arterial digital }\end{array}$ & $\begin{array}{l}\text { Monitorización macrohemodinámica. } \\
\text { Seguridad del test } \\
\text { Variables que orientan a la detención o la } \\
\text { mantención del examen }\end{array}$ \\
\hline Nivel subjetivo de disnea & $\begin{array}{l}\text { Escala de Borg ( } 0-10) \\
\text { ( } 0 \text { es nada, } 10 \text { es máxima dificultad) }\end{array}$ & $\begin{array}{l}\text { Permiten objetivar nivel de disnea al inicio } \\
\text { y término del examen }\end{array}$ \\
\hline $\begin{array}{l}\text { Nivel de cansancio de } \\
\text { extremidades }\end{array}$ & $\begin{array}{l}\text { Escala de Borg ( } 0 \text { - } 10) \\
\text { ( } 0 \text { es nada, } 10 \text { es máxima dificultad) }\end{array}$ & $\begin{array}{l}\text { Permiten objetivar nivel de fatiga o cansan- } \\
\text { cio al inicio y término del examen }\end{array}$ \\
\hline Respiratorias & $\begin{array}{l}\text { Espirometría, curva flujo volumen, } \\
\text { capacidad inspiratoria, ventilación } \\
\text { voluntaria máxima }\end{array}$ & $\begin{array}{l}\text { Estado funcional respiratorio } \\
\text { Reserva respiratoria } \\
\text { Atrapamiento aéreo }\end{array}$ \\
\hline \multirow[t]{5}{*}{ Oxihemodinámicas } & $\begin{array}{l}\mathrm{VO}_{2} \text { o Consumo de oxígeno "peak", } \\
\text { expresado en } \mathrm{mL} / \mathrm{min} \text { o en } \mathrm{mL} / \mathrm{min} / \mathrm{kg} \\
\mathrm{VN}:>80 \% \text { predeterminado }\end{array}$ & $\begin{array}{l}\text { Capacidad de esfuerzo, capacidad aeróbica, } \\
\text { limitación al esfuerzo }\end{array}$ \\
\hline & $\begin{array}{l}\text { Umbral anaeróbico } \\
\text { VN: }>45 \% \mathrm{VO}_{2} \text { max predeterminado }\end{array}$ & Permite estimar capacidad aeróbica \\
\hline & Pulso de $\mathrm{O}_{2}\left(\mathrm{VO}_{2} /\right.$ frecuencia cardíaca $)$ & $\begin{array}{l}\text { Refleja la cantidad de oxígeno extraído por } \\
\text { latido }\end{array}$ \\
\hline & $\begin{array}{l}\text { Pendiente o "slope" entre } \mathrm{VO}_{2} / \mathrm{VCO}_{2} \\
\text { (equivalente respiratorio) } \\
\text { En UL o } \mathrm{VO}_{2} \text { max: } \mathrm{VN}<34 \mathrm{LO}_{2} / \mathrm{min}\end{array}$ & $\begin{array}{l}\text { Se encuentra alterado en toda situación de } \\
\text { alteración V/Q o falla cardiocirculatoria. } \\
\text { Es un indicador de ineficiencia ventilatoria. }\end{array}$ \\
\hline & $\begin{array}{l}\text { Pendiente o slope } \mathrm{VO}_{2} / \mathrm{WR} \text { (relación } \\
\text { consumo/carga de trabajo en watt) } \\
\mathrm{VN}=10\end{array}$ & $\begin{array}{l}\text { Refleja la capacidad de los músculos de } \\
\text { extraer oxígeno y proveer ATP. Nos orienta } \\
\text { a compromiso muscular como limitante del } \\
\text { esfuerzo, desentrenamiento }\end{array}$ \\
\hline
\end{tabular}

VN: valor normal; UL: Umbral láctico. 
Tabla 2. Valor pronóstico de la prueba cardiopulmonar según patología de base ${ }^{3}$

\begin{tabular}{|c|c|c|}
\hline Entidad & $\begin{array}{l}\text { Punto de corte de } \mathrm{VO}_{2} \\
\text { peak }(\mathrm{mL} / \mathrm{kg} / \mathrm{min})\end{array}$ & Comentario \\
\hline Insuficiencia cardíaca & $\begin{array}{l}<14 \\
\mathrm{VE} / \mathrm{VCO}_{2}>40 *\end{array}$ & Indicación para trasplante cardíaco \\
\hline EPOC (enfisema pulmonar) & $<10$ & Factor de mal pronóstico (menor sobrevida) \\
\hline Hipertensión pulmonar & $<10,4$ & Factor de mal pronóstico (riesgo de muerte prematura) \\
\hline $\begin{array}{l}\text { Cirugía de resección } \\
\text { pulmonar }\end{array}$ & $\begin{array}{l}<10 \\
10-15 \\
>15\end{array}$ & $\begin{array}{l}\text { Índice de inoperabilidad } \\
\text { Riesgo elevado } \\
\text { Riesgo aceptable para realizar neumonectomía o } \\
\text { lobectomía }\end{array}$ \\
\hline Fibrosis quísitica & $\begin{array}{l}<14 \text { ó } \\
<58 \% \mathrm{VO}_{2} \text { predeterminado }\end{array}$ & Indicación para realizar trasplante bipulmonar \\
\hline Cirugía bariátrica & $<14,5$ & $\begin{array}{l}\text { Indicador de mayor riesgo de complicaciones después } \\
\text { de la cirugía }\end{array}$ \\
\hline
\end{tabular}

*El equivalente ventilatorio es complementario al valor de $\mathrm{VO}_{2}$ y se ha considerado un indicador fiel de mal pronóstico en Insuficiencia cardíaca.

explicación del test, espirometría, curva flujo/volumen, capacidad inspiratoria) previo al ejercicio en el cicloergómetro que debiera durar entre 8-12 min. Se recomienda realizar pruebas cortas de 10 min en promedio (en la bicicleta) ya que su prolongación lleva a fatiga y término del examen por esta causa y no acorde a su funcionalidad cardiorespiratoria, eludiendo información relevante que puede extraerse de un examen breve, programado acorde con el estado funcional del paciente.

El $\mathrm{VO}_{2}$ obtenido en cicloergómetro es 6-12\% inferior al obtenido en trotadora, sin embargo, se prefiere el cicloergómetro para los pacientes y la trotadora para los deportistas, por motivos de seguridad y estabilidad de los registros. Los exámenes deben durar entre 6-12 min, de preferencia cortos, con una base de calentamiento de al menos un minuto, con 3 minutos de ejercicio a carga baja y luego a cargas incrementales según cada paciente. En general, la carga por intervalos fluctúa entre 5 y 35 watts.

Valor pronóstico del examen. El TCP ha demostrado ser útil para discriminar o direccionar la necesidad de una intervención, terapia o procedimiento. Su beneficio está claramente demostrado en la literatura en pacientes con insuficiencia cardiaca, hipertensión pulmonar, cirugía de resección pulmonar, cirugía bariátrica, EPOC y fibrosis quística (Tabla 2).

Aporte del examen en deportistas. El TCP es de gran utilidad para el seguimiento de los deportistas de alto rendimiento, para deportistas que desean evaluar su capacidad funcional aeróbica, nivel de entrenamiento y estado funcional, lo que les permite estratificarse en diversas categorías según su nivel aeróbico y esto puede ser la condición basal previo a un cambio en el nivel de entrenamiento. El entrenamiento sistemático genera una serie de modificaciones positivas que logran mejorar el $\mathrm{VO}_{2}$ peak, disminuir la taquicardia inducida por el ejercicio y optimizar el umbral láctico. Todo ello a consecuencia de un aumento del gasto cardíaco, una mejoría en la circulación local por aumento de la red capilar muscular y un aumento del número de mitocondrias y enzimas que favorecen un mejor transporte y utilización del oxígeno.

Este examen permite conocer la capacidad aeróbica del deportista de manera objetiva y directa y es de utilidad para planificar estrategias de entrenamiento (carga de trabajo y UA) y como reevaluación de un período de entrenamiento.

Aporte del test cardiopulmonar en la evaluación perioperatoria. El pase operatorio de cirugía resectiva pulmonar es relevante para prever y minimizar la morbimortalidad perioperatoria y no rechazar enfermos oncológicos operables. Se han desarrollado numerosos factores pronósticos y algoritmos de trabajo en este tema logrando algunos elementos de consenso como son que las evaluaciones está- 
ticas basadas en gases arteriales, espirometría y difusión por sí solas no son suficientes, y si bien se considera de mayor riesgo un $V E F_{1}<40 \%$, un $V E F_{1}$ postoperatorio predicho $<33 \%$, una $\mathrm{PCO}_{2}>45$ $\mathrm{mmHg}$, el test cardiopulmonar ha permitido distinguir con mayor precisión los pacientes de mayor riesgo quirúrgico $\left(\mathrm{VO}_{2}\right.$ peak $<10 \mathrm{ml} / \mathrm{kg} / \mathrm{min}$ ó $<35 \%$ del predictivo $\left.{ }^{4,5}\right)$. El algoritmo publicado en 2009 por la European Respiratory Society ${ }^{6}$ expresa muy claramente la utilidad del test cardiopulmonar en etapas precoces de la evaluación de los pacientes candidatos a cirugía resectiva.

Dra. Mónica Zagolín B.

Coordinadora $31^{a}$ Jornadas de Otoño, Sociedad Chilena de Enfermedades Respiratorias Depto. Medicina, Facultad de Medicina, Universidad de Chile, Instituto Nacional del Tórax Medicina Interna y Broncopulmonar, Clínica Santa María, Santiago-Chile E-mail: monizagolin@hotmail.com

\section{Referencias bibliográficas}

1.- ATS/ACCP Statement on Cardiopulmonary Exercise Testing. Am J Respir Crit Care Med 2003, 167: 211-77.

2.- BALADY G J, ARENA R, SIETSEMA K, MYERS J, COKE L, FLETCHER G F, et al. Clinician's guide to cardiopulmonary exercise testing in adults: a scientific statement from the American Heart Association. American Heart Association Exercise, Cardiac Rehabilitation, and Prevention Committee of the Council on Clinical Cardiology; Council on Epidemiology and Prevention; Council on Peripheral Vascular Disease; Interdisciplinary Council on Quality of Care and Outcomes Research. Circulation 2010; 122: 191-225.

3.- PALANGE P, WARD S A, CARLSEN K H, CASABURI R, GALLAGHER C G, GOSSELINK R, et al. Recommendations on the use of exercise testing in clinical practice. ERS Task Force. Eur Respir J 2007; 29: 185-209.

4.- COLICE G, SHAFAZAND S, GRIFFIN J, KEENAN R, BOLLIGER C. Physiologic evaluation of the patient with lung cancer being considered for resectional surgery: ACCP Evidenced-Based Clinical Practice Guidelines (2nd Edition). Chest 2007; 132: 161S-77S.

5.- BRUNELLI A, CHARLOUX A, BOLLIGER C T, ROCCO G, SCULIER J P, VARELA G, et al. The European Respiratory Society and European Society of Thoracic Surgeons clinical guidelines for evaluating fitness for radical treatment (surgery and chemoradiotherapy) in patients with lung cancer. Eur J Cardiothorac Surg 2009; 36: 181-4.

6.- ERS/ESTS. Clinical guidelines on fitness for radical therapy in lung cancer patients. Eur Respir J 2009; 34: 17-41. 